\title{
An observational evidence for the Babcock-Leighton dynamo scenario
}

\author{
D. V. Erofeev \\ Ussuriysk Astrophysical Observatory, 692533 Gornotaeznoe, Russia \\ email: erofeev@utl.ru
}

\begin{abstract}
In order to verify scenario of solar cycle suggested by Babcock and Leighton, a new index of activity is derived from observational data of bipolar spot groups by taking positions of magnetic poles of the groups into account. In agreement with the Babcock-Leighton scenario, this index reveals substantial correlation with amplitude of the axisymmetric magnetic field of the Sun measured at subsequent minimum of activity.
\end{abstract}

According to the Babcock-Leighton dynamo scenario, the poloidal magnetic field of the Sun is a product of decay and diffusion of large active regions associated with bipolar spot groups (Leighton 1964). This permits to expect that there is a relationship between sunspot activity in an 11-yr cycle and intensity of the large-scale axisymmetric magnetic field observed at the subsequent cycle minimum. However, such indices of activity as spot number or total area of sunspots are useless to derive this relationship because efficiency of a bipolar spot group as a source of the poloidal magnetic field depends on positions of its magnetic poles. Also, effect of the meridional plasma flow on horizontal transport of magnetic flux should be taken into account (Wang, Nash \& Sheeley 1989).

In order to describe the contribution made by bipolar spot groups to the poloidal magnetic field during $n$-th cycle of activity, I introduce index $d_{n}$ as follows:

$$
d_{n}=\sum_{i} g\left(f_{i}\right) S_{i} \Delta f_{i} \cos \left(f_{i}\right)
$$

In Equation (0.1), the sum includes bipolar spot groups of $n$-th cycle, $S_{i}$ and $f_{i}$ are maximum observed area and latitude of the center of $i$-th group, while $\Delta f_{i}$ is distance in latitude between its leading and following magnetic poles $\left(\Delta f_{i}>0\right.$ in case the leading pole of the group lies more closely to the equator than the following one, and $\Delta f_{i}<0$ in an opposite case). The weighting function, $g(f)$, takes meridional transport of magnetic flux into account. Qualitative analysis and flux transport simulations both indicate that $g(f)$ should decrease with increasing $|f|$ due to effect of the poleward directed meridional flow. In practice, I use the expression $g(f)=\exp \left[-(f / F)^{2}\right]$, however, parameter $F$ cannot be determined from the simulations with due accuracy because the diffusion constant and meridional flow velocity are known only approximately. An appreciable estimate of $F$ has been found from analysis of the observational data (see below).

In order to derive a time series of $d_{n}$, I use data of bipolar spot groups obtained at Kodaikanal Observatory (KO) in years 1906-1987, at Mount Wilson Observatory (MWO) in years 1917-1985 (see Howard 1991), and at Ussuriysk Astrophysical Observatory (UAO) during the period from 1956 to 1996 (see Erofeev 2001). The data of KO and MWO have been obtained from NGDC web site. Values of $d_{n}$ separately derived from the three data sets have been reduced to same scale and then averaged.

For comparison with index $d_{n}$, I use amplitude of the axisymmetric magnetic dipole of the Sun, D, inferred from a series of the H-alpha synoptic maps (see Makarov et al. 2001). 


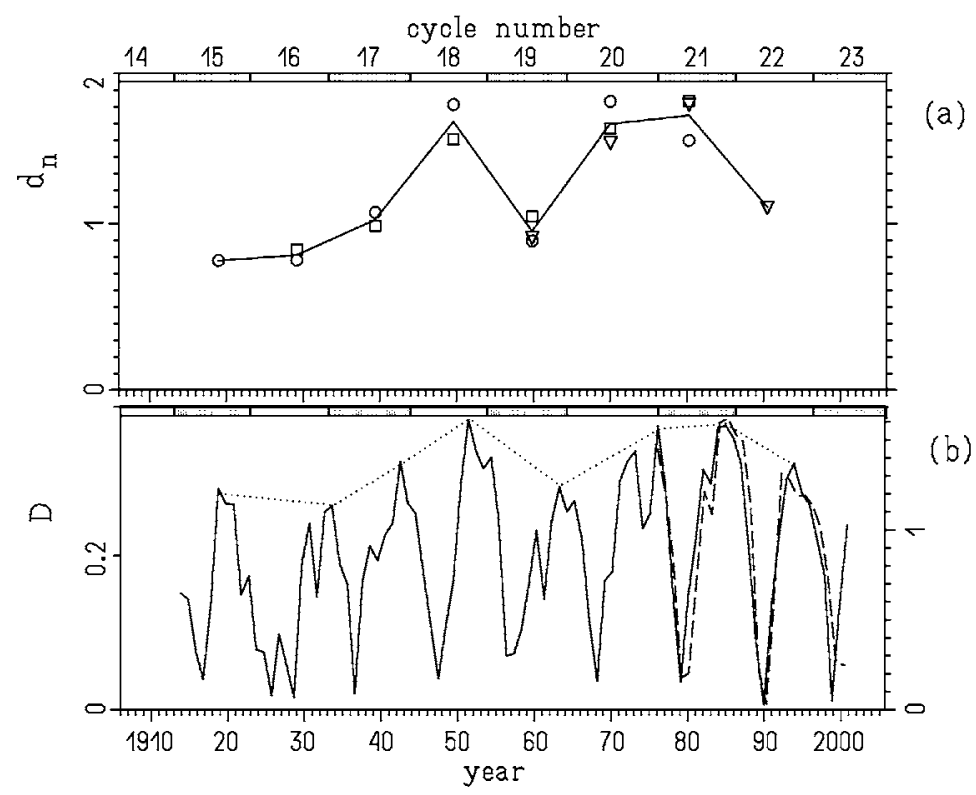

Figure 1. (a): Index $d_{n}$ which describes the contribution made by bipolar spot groups to the axisymmetric dipole of the Sun. Values of $d_{n}$ separately calculated from data of three observatories are indicated by circles (KO), rectangles (MWO), and triangles (UAO), while solid line indicates the averages. (b): Amplitude of the axisymmetric dipole of the Sun, $D(t)$, derived from the H-alpha data (solid line and left scale) and from WSO synoptic maps of magnetic field (long-dashed line.)

Each 11-yr cycle is characterized by maximum annual value of the dipole amplitude, $D_{n}$ (emphasize that $D_{n}$ corresponds to the activity minimum following $n$-th cycle of sunspots).

I applied correlation analysis to investigate the relationship between index $d_{n}$ and amplitude of the axisymmetric solar dipole, $D_{n}(n=15 . .22)$. An appreciable estimate of the unknown parameter $F$ has been found as the value that provided a maximum correlation between $d_{n}$ and $D_{n}$. This yields $F=9.5^{\circ}$, and the maximum value of the correlation coefficient $r\left(d_{n}, D_{n}\right)=0.96$. On the other hand, no correlation has been found between $d_{n}$ and dipole amplitude measured at preceding minimum of activity, $D_{n-1}$.

Figure 1 exhibits a substantial agreement between $d_{n}$ and amplitude of the axisymmetric dipole of the Sun measured at subsequent minimum of activity. This agreement gives evidence for the Babcock-Leighton scenario of regeneration of the poloidal magnetic field. Note also that index $d_{n}$ substantially correlates with maximum spot number in the next cycle, $R_{n+1}: r\left(d_{n}, R_{n+1}\right)=0.85$. Thus, $d_{n}$ may be used to forecast the level of activity in the next 11-yr cycle.

\section{References}

Erofeev, D. V. 2001 Solar Phys. 198, 31-50.

Howard, R. 1991 Solar Phys. 136, 251-262.

Leighton, R. B. 1964 Astrophys. J. 140, 1547-1562.

Makarov, V. I., Tlatov, A. G., Callebaut, D. K., Obridko, V. N. \& Shelting, B. D. 2001 Solar Phys. 198, 409-421.

Wang, Y.-M., Nash, A. G. \& Sheeley, N.R. 1989 Science 245, 712-718. 